\title{
Research Progress of Grain Quality Nondestructive Testing Methods
}

\author{
Fenghua Wang ${ }^{* *}$, Zhiyong Xi, Ju Yang, and Xiaojing Yang \\ Faculty of Modern Agricultural Engineering, Kunming University of Science and Technology, \\ Kunming, Yunnan, China 650500 \\ wangfenghua018@163.com
}

\begin{abstract}
The application of modern non-destructive testing methods on grain quality testing had important implications for grain production, distribution and sale. In the paper, five test methods of modern electronic information technology, which were near-infrared spectroscopy, electronic nose, machine vision, magnetic resonance and acoustic vibrations, were described, the research progress of near-infrared spectroscopy, electronic nose technology and machine vision technology in grain quality nondestructive testing was reviewed with focuses. The application of modern non-destructive testing methods on grain quality testing could reduce costs and improve detection accuracy, furthermore, make it combined with modern computer technology and data processing, which would play an important role in agriculture.
\end{abstract}

Keywords: Grain, Quality, Non-destructive testing, Research process.

\section{Introduction}

Cereal is a traditional diet of Chinese and an important and indispensable food of human survival [1]. Rice annual production of our country is 200 million tons, accounting for 35 percent of world output. Most of the food needs circulation and warehouse storage. However, in domestic and foreign trade and the circulation process of grain, food quality testing methods of many grain purchasing department rely on the person's subjective assessment because the lack of rapid quality detection means, which is not only can not detect a variety of internal quality parameters of food, and there is a great error. Using modern non-destructive testing method to identify the grain quality can reduce costs and improve the detection accuracy, China's grain quality testing technology research and application levels will be improved [2].

\footnotetext{
* This study was financed by the Application Basis Surface Projects of Yunnan Province (2010ZC028), Kunming University of Science and Technology Analysis and Testing Fund (2010299).

** Corresponding author.
}

D. Li and Y. Chen (Eds.): CCTA 2012, Part II, IFIP AICT 393, pp. 255-262, 2013.

(C) IFIP International Federation for Information Processing 2013 
Non-destructive testing (NDT) is general term for all the technical means to test the existence of different degrees of the defects in the detected objects, give out the location, size, number and nature of the defect, determine the technical state (such as eligibility, remaining life, etc) of the detected objects, using sound, light, electricity, magnetism, taste and other characteristics, under the premise that does not damage or affect the performance of the detected objects [3]. Modern non-destructive testing methods (such as near-infrared spectroscopy, the electronic nose and machine vision technology, nuclear magnetic resonance and acoustic vibrations method) are mainly based on the density, hardness, shape, color and other parameters of the detected objects, to determine the quality of agricultural products such as maturity, sugar and acid ratio, moisture content, sugar content, internal lesions and so on, which are both to ensure the integrity of the sample and can effectively determine the internal quality information [4]. In the paper, five test methods of modern electronic information technology, which were near-infrared spectroscopy, electronic nose, machine vision, magnetic resonance and acoustic vibrations, were described. The research progress of the near-infrared spectroscopy, electronic nose and machine vision technology in the non-destructive testing of grain quality were reviewed mainly.

\section{Near-Infrared Spectroscopy and the Application Status in the Grain Non-destructive Testing}

Near Infrared Spectroscopy (NIRS) is electromagnetic waves that the wavelength is in the 780-2526nm between visible light and infrared light [5].Due to composing frequency and all levels double frequency absorption of containing hydrogen group vibration in organic molecules was consistent with the near infrared region, therefore, vibration information of containing hydrogen group in the sample organic molecules can be obtained by scanning with near infrared spectroscopy [6]. Compared with traditional methods of chemical analysis, the main technical characteristics of the near-infrared spectroscopy are fast analysis, simultaneous determination of multicomponent samples without pretreatment, non-destructive analysis, distance measurement and real-time analysis, low analysis cost and simple operation. So the near-infrared spectroscopy is known as the giant of the analyst community [7].

In the $1960 \mathrm{~s}$, Norris determined the moisture, protein, and fat content of the grains using near-infrared spectroscopy and multivariate linear regression analysis. Later, Hymowitz uses near infrared technology for the determination of protein, fat, and amino acid content of the cereal crops. Kong Wenwen et al. [8] detected malondialdehyde (MDA) content in the barley leaves using spectroscopy and have high precision. a new method for status monitoring of physiological information about the large scale agriculture of hordeum growth and stress of herbicide has been provided. Xie Xinhua et al. [9] established a near-infrared mathematical model of the single-grain brown rice protein which improves the near-infrared quantitative analysis with high accuracy. Zhu Wenchao et al. [10] applied visible / NIR spectroscopy to realize the identification of the transgenic rice leaves and detection of 
the chlorophyll content quickly. A new method for the realization of the living identification and continuous monitoring in field has been offered.

The grain protein distribution has been studied by Engel et al. [11]. The predictive model of spring wheat has been established under the laboratory conditions. the online prediction of the protein concentration of spring wheat in farmland by the model, The results showed that the results of distribution of grain protein content by online and laboratory are very similar, the correlation coefficient is 0.93 . Lu Hui et al. [12] established the method of the fast species identification and eating quality analysis on rice by near infrared spectroscopy. Near infrared spectrum of 102 crushed rice samples have been collected, the quantitative analysis model of water, protein and amylose on rice by partial least squares (PLS) method, to evaluate the accuracy of the predicted results. The results show that NIRS analysis techniques can be used for rapid non-destructive testing of the quality and variety of rice. Liu Xiaojun et al. [13] found that it had a obvious spatial and temporal variation characteristics of leaf reflectance spectrum in the different soil water and leaves, and sensitive spectral bands of leaf water content located in the near infrared and shortwave infrared region, the spectral index and leaf water content showed a good linear correlation, the linear fitting R2 of 0.80 . Therefore, a rapid non-destructive diagnosis of rice leaf water content estimation model has been established to provide the basis for the precise management of rice water. Sun Xiaorong et al. [14] have detected the water content of starch by near infrared reflectance spectroscopy, at first the different types of starch at home and abroad have been collected, then the optimal pretreatment methods and spectral range have been selected, finally quantitative analysis of the water content of starch by partial least squares method, The results showed that rapid non-destructive testing of starch water content using near-infrared spectroscopy is available.

\section{Electronic Nose Technology and the Application Status in Grain Non-destructive Testing}

The electronic nose is an electronic system of the simulating biological nose to analyze and detect complex odor and volatile chemical composition mainly. The electronic nose is a kind of instrument can identify a variety of odor, which composed of the selective chemical sensor arrays and the appropriate pattern recognition system. Generally it consists of three parts of the gas sensor array, signal processing unit and the pattern recognition unit. On working, the signal is generated after the adsorption of odor molecules by the gas sensor array, then the signal is sent to the signal processing unit for processing, and finally the analysis results is obtained by the pattern recognition unit analyzing the data of signal processing $[15,16]$.

On mouldy detection aspect, Zou Xiaobo et al. [17] developed a rapid detection grain whether moldy electronic nose device, the device can be fast, accurate analysis of the measured grain odor, and its correct rate is $90 \%$ or more. Pan Tianhong et al. [18-19] developed a group of thick-film metal oxide tin gas sensors (MOS) array in the identification of moldy corn. The data of the entire reaction process between the sensor and the grain volatile odors, the characteristic value of the sensor and the odor 
response have been extracted, and have been analyzed by RBF neural network, the result show that the back estimation rate reached $100 \%$ and the recognition probability is $92.19 \%$. On the insect detection aspect, Ridgway et al. [20] detected the insect infected wheat samples using the electronic nose, the feasibility of the method has been proved. Jiang Deyun et al. [21] designed test system using the electronic nose to test and compare the gas samples of containing live worms, containing dead insects and standard, then the pattern recognition have been carried on by principal component analysis. The result shows that this method can quickly detect the pests' erosion of food whether or not. Zhang Hongmei et al. [22] detected the pest wheat and the mixture of the pest wheat and normal wheat on the 5storage period by electronic nose. The results show that the pest wheat and normal wheat and mixed with different proportions of insect pests of wheat samples can be detected by this method. On the storage year detection aspect, Pang Lingjiang et al.[23,24] distinguished the five year wheat using portable electronic nose, The results show that the principal component analysis can identify the different years of wheat, the recognition accuracy is up to $85 \%$ by BP neural network analysis. Zhang Hongmei et al. [25] identified the wheat of the different storage life using electronic nose, and sensor array signal has been optimized, the BP neural network model has been established using the optimized sensor, and the recognition rate is up to $96 \%$.

\section{$4 \quad$ Machine Vision Technology and the Application Status in Grain Non-destructive Testing}

Machine vision technology is a kind of technology using imaging sensor instead of the human eye to obtain the object image, which converts images into digital information to analyze the images and draw a conclusion, and carry on nondestructive testing to internal and external quality of an agricultural products, such as the shape, size, color, surface defects and the maturity [26].

Huang Xingyi et al. [27] identified the rice varieties by the computer vision technology instead of human eye. The method that has combined the shape with the color characteristics of the image to identify the rice varieties has been proposed. The recognition classifier has been established by Bayesian decision method, and the recognition accuracy can reach $88.3 \%$. Casady et al. [28] collected the image of the paddy rice crown using the machine vision systems, the image segmentation and feature extraction have been carried on by gray-scale median values and mathematical morphology. The study found that the correlation of height, width and area of rice crown measured by the system and manual are $0.896,0.874$ and 0.885 respectively, which has provided decision-making support for rational application of nitrogen fertilizer. R. Choudhary et al. [29] extracted the shape, color, texture and wavelet feature of wheat from the color image, the classification accuracy of wheat varieties have been compared using the linear classifier and the quadratic classifier. Combining with the different number of wheat characteristics, the results of the classification show that the detection results combined with wheat shape, color, texture, and wavelet four kinds of characteristics are the best. Aiming at the segmentation 
problems of a large number of cereal grain adhesion image, Lin Yun et al. [30] improved the erosion and dilation algorithms and watershed segmentation algorithm based on morphological. The relative error is less than $2 \%$.

\section{Nuclear Magnetic Resonance and Acoustic Vibration}

Nuclear magnetic resonance (NMR) is one of the technologies to detect concentrated hydrogen [31], which is sensitive to respond changes of the agricultural water and lipid in the mixed granulating state. Research object of nuclear magnetic resonance is the nucleus having the magnetic moments. In the static magnetic field, this has a magnetic moment nucleus exist the different energy levels. The samples are radiated by a particular frequency of electromagnetic radiation, the transition between energy level of the nucleus will be carried on, which is nuclear magnetic resonance.

Loveday et al.[32] studied the physical and chemical changes model of the protein bar in the store process of the 50 day in $20^{\circ} \mathrm{C}$, the study discover that the chemical works is very small in the protein hardening process, mainly due to moisture migration caused by micro-structural changes and molecular activity of impact. Vanlent et al.[33] determined the small water droplet size and distribution of the six different fabrication of butter using the NMR and confocal laser scanning microscopy to ensure the microbial stability and sensory quality. The results show that the different fabrication of butter put up the noticeably different. In recent years, NMR techniques have been widely used in medicine [34], which plays an important role in the medical examination and biochemical research, but rarely used in agricultural products. With the low field strength, precision, low-cost, high-speed research and development of NMR technology, NMR technology has great potential and will play an important role in the agricultural domain [35].

The acoustic vibration is a means of method to determine the quality of the detected object according to mechanical vibration measurement results that is produced through an incentive to the test specimen. The characteristic of the acoustic vibration is simple, rapid, and inexpensive [36].

Knocking detection is one of the most simple and commonly method used as nondestructive testing techniques relying on the component vibration characteristics [37]. Acoustic vibration detection methods should be further studied from the theory and practice, the acoustic vibration detection methods of easy operation, convenient and reliable would be developed, which will further promote the practical application of acoustic vibration detection technology. On the food safety monitoring aspect, Nassar et al.[38] inspected the dynamics formation process of milk floc using low-energy ultrasonic resonance testing technology, the influence of raw milk temperature and chymosin concentration to milk flocculation have been analyzed. Elvira L et al. [39] studied found that the ultra high temperature sterilization packaged milk has been detected using $800 \mathrm{KHz}$ ultrasonic to determine the changes of the ultrasonic propagation time and amplitude, which can detect the growth of microorganisms in liquid food. Gan T H et al.[40] proved that packaging products can be detect by the non-contact method, even including the detection of liquid with starch as a raw 
material in microwave packaging contain using air-coupled ultrasonic technology to detect liquid and starch-based raw materials in the container.

\section{Summary}

Although near-infrared spectroscopy has the advantages of fast, easy, simple, accurate and the simultaneous determination of multi-component samples in the online analysis, it is an indirect analysis technique and still has many shortcomings. The development direction of near infrared spectroscopy technology is the imaging spectroscopy. With the development of two-dimensional array detector, the measurement of composition and component distribution become possible. The electronic nose use as an electronic olfactory sensor system, shown unique features in the food, quality control, quality evaluation and safety testing, Which combined with the parallel coupled system of gas chromatography instrument and flame ionization detector become a new trend. Machine vision technology application in agriculture is still in the initial stage, the agricultural product range is limited, and most used in quality detection of agricultural products. And now, development of the agricultural harvest and processing automation field should be to meet the requirements of increasing of the harvesting and processing automatic degree of the agricultural products and improving of the production efficiency, should be further strengthened to speed up research in the field. NMR techniques played an important role in the medical examination and biochemical fields, but agriculture is still a new field, should increase the strength on cereal and fruit application respect in the future. Acoustic vibration detection methods should be further studied from the theory and practice, to deep study of acoustic vibration testing methods of grain, and establish the relationship between signals of sound vibration and defects. The portable mensuration instrument will become the development trend of the sound vibration technology.

\section{References}

1. $\mathrm{Wu}, \mathrm{S}$., Cheng, Z.: Grain storage and quality maintenance. Chinese Journal of Animal Science 46(6), 52-54 (2010) (in Chinese)

2. Yu, Z., Hou, Y.: Development of grain ventilation drying quality. Drying Technology and Equipment 7(1), 41-44 (2009) (in Chinese)

3. Li, J., Rao, X., Ying, Y.: Advance on Application of Hyperspectral Imaging to Nondestructive Detection of Agricultural Products External Quality. Spectroscopy and Spectral Analysis 31(8), 2021-2026 (2011) (in Chinese)

4. Sun, T., Xu, H., Ying, Y.: Progress in Application of Near Infrared Spectroscopy to Nondestructive On-line Detection of Products/Food Quality. Spectroscopy and Spectral Analysis 29(1), 122-126 (2009) (in Chinese)

5. Xu, X., Cheng, F., Ying, Y.: Application and Recent Development of Research on NearInfrared Spectroscopy for Meat Quality Evaluation. Spectroscopy and Spectral Analysis 29(7), 1876-1880 (2009) (in Chinese)

6. Blanco, M., Villarreal, I.: NIR spectroscopy rapid-response analytical tool. Trends in Analytical Chemistry 21(4), 240-250 (2002) 
7. Gong, Y., Zhang, W.: Recent Progress in NIR Spectroscopy Technology and Its Application to the Field of Forestry. Spectroscopy and Spectral Analysis 28(7), 1544-1548 (2008) (in Chinese)

8. Kong, W., Liu, F., Hui, F., et al.: Rapid detection of malondialdehyde in herbicide-stressed barley leaves using spectroscopic techniques. Transactions of the Chinese Society of Agricultural Engineering 28(2), 171-175 (2012) (in Chinese)

9. Xie, X., Xiao, X., Li, F., et al.: Mathematic Models for Analyzing Protein Content in Single Brown Rice with Near Infrared Spectroscopy. Transactions of the Chinese Society for Agricultural Machinery 37(8), 120-122 (2006) (in Chinese)

10. Zhu, W., Cheng, F.: Analysis of Transgenic and Non-Transgenic Rice Leaves Using Visible/Near-Infrared Spectroscopy. Spectroscopy and Spectral Analysis 32(2), 370-373 (2012) (in Chinese)

11. Engel, R.E., Long, D.S.: Measuring grain protein concentration with in-line near infrared reflectance spectroscopy. Crop Management 10(5), 1-3 (2005)

12. Lv, H., Zhang, Z., Wang, S.P., et al.: Quality analysis and category identification of rice based on the near infrared spectroscopy (NIRS). Science and Technology of Food Industry 33(3), 322-325 (2012) (in Chinese)

13. Liu, X., Tian, Y., Yao, X., et al.: Monitoring Leaf Water Content Based on Hyperspectra in Rice. Scientia Agricultura Sinica 45(3), 435-442 (2012) (in Chinese)

14. Sun, X., Liu, C., Wu, J., et al.: Rapid detection of water content in starch based on near infrared spectrum. Science and Technology of Food Industry 32(10), 441-442 (2011) (in Chinese)

15. Zhang, H., He, Y.: The Applications of Electronic Nose in the Grain Quality Discrimination. Journal of Agricultural Mechanization Research 15(3), 180-182 (2009) (in Chinese)

16. Wei, L., Zhang, C., Zhao, B., et al.: Electronic Nose Technology and Its Application in the Activity Detection of Wheat Grain. Journal of Agricultural Mechanization Research 9(6), 150-152, 196 (2010) (in Chinese)

17. Zou, X., Zhao, J.: Rapid identification of moldy corn by electronic nose. Transactions of the CSAE 20(4), 121-124 (2004) (in Chinese)

18. Pan, T., Chen, S., Zhao, D.: Application of Electronic Nose Technology in Moldy Foodstuff Recognition. Instrument Technique and Sensor 3, 51-52 (2005) (in Chinese)

19. Zhu, J., Zhao, D., Pan, T., et al.: Identification of moldy foodstuff based on artificial olfactory system. Transactions of the CSAE 21(1), 106-109 (2005) (in Chinese)

20. Hu, J., Guo, M., Zhang, J., et al.: New detection technology and application status of stored-grain insects. Transactions of the CSAE 23(11), 286-290 (2007) (in Chinese)

21. Jiang, D., Kong, X., Li, B., et al.: Research and Application of Electronic Nose for Testing Stored-grain Insects. Journal of Anhui Agricultural University 32(2), 254-257 (2005) (in Chinese)

22. Zhang, H., Jun, W.: Detection of age and insect damage incurred by wheat, with an electronic nose. Journal of Stored Products Research 43, 489-495 (2007)

23. Pang, L.: Application of electronic nose in identification of wheat freshness. Zhejiang University, Hangzhou (2005)

24. Pang, L., Wang, J., Lu, X.: Discrimination of Different Storage Time of the Wheat by Electronic Nose. Chinese Journal of Sensors and Actuators 20(8), 1717-1722 (2007) (in Chinese)

25. Zhang, H., Wang, J., Tian, X., et al.: Optimization of sensor array and detection of stored duration of wheat by electronic nose. Journal of Food Engineering 82(6), 403-408 (2007) 
26. Ying, Y., Zhang, W., Jiang, Y., et al.: Application of Machine Vision Technique in Automatic Harvesting and Processing of Agricultural Products. Transactions of the Chinese Society for Agricultural Machinery 31(3), 112-115 (2000) (in Chinese)

27. Huang, X., Li, J., Jiang, S.: Study on identification of rice varieties using computer vision. Journal of Jiangsu University (Natural Science Edition) 25(2), 102-104 (2004) (in Chinese)

28. Casady, W., Singh, N., Costello, T.A.: Machine vision for measurement of rice caopy dimensions. Trans. of the ASAE 39(5), 1891-1898 (1996)

29. Choudhary, R., Paliwal, Jayas, D.S.: Classification of cereal grains using wavelet, morphological color, and textural features of non-touching kernel images. Biosystems Engineering 99(3), 330-337 (2008)

30. Ling, Y., Wang, Y., Sun, M., et al.: Application of Watershed Algorithm to Paddy Image Segmentation. Transactions of the Chinese Society for Agricultural Machinery 36(3), 9598 (2005) (in Chinese)

31. Zhou, S., Shang, D., Ying, Y., et al.: Detecting Subtle Bruises on Fruits with Nuclear Magnetic Resonance Imaging. Transactions of the Chinese Society for Agricultural Machinery 41(8), 107-110 (2010) (in Chinese)

32. Loveday, S.M., Hindmarsh, J.P., Creamer, L.K., et al.: Physicochemical changes in a model protein bar during storage. Food Research International 42(7), 798-806 (2009)

33. Vanlent, K., Vanlerbergbe, B., Oostreldt, P.V., et al.: Determination of water droplet size distribution in butter: pulsed field gradient NMR in comparison with confocal scanning laser microscopy. International Dairy Journal 18(1), 12-22 (2008)

34. Pang, L., Wang, Y., He, Z., et al.: Application of NMR in Fruit Quality Detection. Journal of Agricultural Mechanization Research (8), 176-180 (2006) (in Chinese)

35. Jin, Z., Zhang, J., Qian, F., et al.: Study on Changes of Grains during Storage by NMR. Food Science 29(9), 66-69 (2008) (in Chinese)

36. Chen, J.: Non-destructive test for the quality of agricultural product. Journal of Changchun University of Technology (Natural Science Edition) 27(3), 262-266 (2006) (in Chinese)

37. Cawley, P., Adams, R.D.: The mechanics of the coin-tap method of non-destructive testing. Journal of Sound Vibration 122(9), 299-316 (1988)

38. Nassar, G., Nongailland, B., Noel, Y.: Monitoring of milk gelation using low-frequency ultrasonic technique. Journal of Food Engineering 48, 351-359 (2001)

39. Elvira, L., Sampedro, F., Montero de Espinosa, et al.: Eight-channel ultrasonic device for non-invasive quality evaluation in packed milk. Ultrasonic 45, 92-99 (2006)

40. Gan, T.H., Hutchins, D.A., Billson, D.R.: Preliminary studies of a novel air-coupled ultrasonic inspection system for food containers. Journal of Food Engineering 53(4), $315-323$ (2002) 